erhitzt Clark nach dem Vorgang von W. Odling*) $100 \mathrm{~g}$ Späne mit einer starken, salzsauren Lösung von Eisen- oder Kupferchlorid ***) in einem geeigneten Destillationsapparate und führt durch wiederholtes Zufügen von starker Salzsäure und erneute Destillation das Arsen vollständig in das Destillat über.

\title{
Zur volumetrischen Bestimmung von Phosphor in Eisen und
} Stahl. Edward D. Camp bell***) gründet eine Methode auf die Thatsachen, dass Molybdänsäure in salzsaurer Lösung durch Zinnchlorür zu Molybdänpentachlorid reducirt wird, und dass man weiter Molybdänpentachlorid maassanalytisch mit Hülfe einer Lösung von Kaliumdichromat bestimmen kann, indem man einen Ueberschuss der letzteren mittelst Eisenchlorürlösung zurücktitrirt. Die Normallösungen werden von dem Verfasser unter der Voraussetzung hergestellt, dass der Niederschlag von phosphormolybdänsaurem Ammon die Zusammensetzung hat

$$
3\left(\mathrm{NH}_{4}\right)_{2} \mathrm{O}, \mathrm{P}_{2} \mathrm{O}_{5}, 22 \mathrm{MoO}_{3}, 12 \mathrm{H}_{2} \mathrm{O} \text {, }
$$

demnach $1,685 \%$ Phosphor und 86,01 \% Molybdänsäure enthält.

Ist eine Phosphorbestimmung in einem Stahl oder Schmiedeeisen auszuführen, so verfährt man folgendermaassen :

$1 \mathrm{~g}$ wird in einem Becherglase in $20-25 \mathrm{cc}$ Salpetersäure von 1,20 specifischem Gewicht gelöst und die Lösung auf einer Eisenplatte auf die Hälfte etwa eingekocht. Nach Zusatz von $10 \mathrm{cc}$ Salpetersäure von dem specifischen Gewicht 1,42 setzt man das Kochen fort und fügt ungefähr $0,5 \mathrm{~g}$ chlorsaures Kali nach und nach zu, bis Mangansuperoxyd sich ausgeschieden hat. Man gibt nun einige Tropfen Salzsäure von 1,19 specifischem Gewicht zu und zerstört so den Ueberschuss an chlorsaurem Kali, während man gleichzeitig das ausgeschiedene Mangansuperoxyd löst. Nach nochmaligem Zufügen von $5 c c$ Salpetersäure von 1,42 specifischem Gewicht kocht man wieder auf $10-15 c c$ ein.

Man setzt jetzt $10-15 c c$ Wasser zu, kühlt $a b$, füllt das Eisenoxyd mittelst Ammoniakflüssigkeit von 0,92 specifischem Gewicht und löst den Niederschlag wiederum in wenig überschüssiger Salpetersäure von 1,42 specifischem Gewicht. Durch die Bildung von salpetersaurem Eisenoxyd und salpetersaurem Ammon wird die Temperatur der Flüssigkeit auf $60-80^{\circ} \mathrm{C}$. gesteigert, und fällt man nun unter Umschütteln mit $20 c c$ einer Molybdän-

*) Diese Zeitschrift 2, 388 .

**) $200 \mathrm{~g}$ Eisen oder Kupfer enthaltend.

***) Journal of analytical chemistry 1, 370 . 
säurelösung, deren Bereitung unten folgt*). Nach 10 Minuten langem Stehen bei $60^{\circ} \mathrm{C}$. hat sich der Niederschlag abgesetzt und die überstehende Flüssigkeit ist klar. Man sammelt jetzt den Niederschlag auf einem Filter, wäscht denselben unter Zuhülfenahme einer Pumpe mit Wasser aus, welches $3 \%$ Salpetersäure von 1,20 specifischem Gewicht enthält, und entfernt schliesslich die Salpetersäure durch zweimaliges Nachwaschen mit Wasser. Der Niederschlag wird hierauf in Ammoniak gelöst und die Lösung sammt Waschwasser in ein Becherglas gebracht und eventuell auf 70-80 cc verdünnt.

- Zu dieser Lösung fügt man zunächst $15 \mathrm{cc}$ Salzsäure von 1,19 specifischem Gewicht, dann 6-8 Tropfen einer Zinnchlorürlösung und prüft endlich, ob auf Zusatz eines weiteren Tropfens der Zinnchlorürlösung keine Veränderung der erst dunkel, später licht braunen Färbung der Flüssigkeit eintritt. Man fügt nun $10 c c$ einer 7 procentigen Quecksilberchloridlösung $\mathrm{zu}$, rührt gut um und lässt aus einer Bürette Kaliumdichromatlösung, von welcher $1 c c 0,0001 \mathrm{~g}$ Phosphor entspricht, einfliessen, bis ein geringer Ueberschuss vorhanden ist**). Nachdem mail jetzt durch Zufügen von $10 c c$ Ammoniak von 0,92 specifischem Gewicht den Salzsäureüberschuss abgestumpft hat, titrirt man mittelst einer Eisenchlorürlösung zurück, von welcher $1 c c 0,0001 \mathrm{~g}$ Phosphor entspricht, bis sich ein schwacher Ueberschuss durch Tüpfeln mit Ferridcyankalium nachweisen lässt, welchen man wiederum durch einige Tropfen Kaliumdichromatlösung wegnimmt.

Liegt ein Roheisen vor, so löst man $1 g$ in einer Porzellanschale in Salpetersäure von 1,2 specifischem Gewicht. Nachdem die Reaction beendet ist, fügt man $5 c c$ Salzsäure von 1,19 specifischem Gewicht zu, kocht ein, trocknet schliesslich vorsichtig aus und erhitzt, bis sich keine sauren Dämpfe mehr wahrnehmen lassen. Man nimmt nun unter Erwärmen mit $10 \mathrm{cc}$ Salzsäure von 1,19 specifischem Gewicht auf, fügt Wasser zu und filtrirt. Filtrat und Waschwasser kocht man unter $\mathrm{Zu}$ satz von $10 c c$ Salpetersäure von 1,42 specifischem Gewicht auf $10-15 c c$ ein und verfährt dann weiter bezüglich des Verdünnens und der Fällung mit Ammoniak wie oben angegeben.

Die bei Ausführung der Methode in Betracht kommenden Reactionen sind nachstehende:

*) $20 \mathrm{ce}$ der Molybdänsäurelösung reichen noch bei $0,15 \%$ Phosphor aus.

**) Bei Bessemerstahl genügen 12-15cc. 
$2 \mathrm{MoO}_{3}+\mathrm{SnCl}_{2}+12 \mathrm{HCl}=2 \mathrm{MoCl}_{5}+\mathrm{SnCl}_{4}+6 \mathrm{H}_{2} \mathrm{O}$. $\mathrm{SnCl}_{2}+2 \mathrm{Hg} \mathrm{Cl}_{2}=\mathrm{SnCl}_{4}+\mathrm{Hg}_{2} \mathrm{Cl}_{2}$. $6 \mathrm{MoCl}_{5}+\mathrm{K}_{2} \mathrm{Cr}_{2} \mathrm{O}_{7}+11 \mathrm{H}_{2} \mathrm{O}=6 \mathrm{MoO}_{3}+\mathrm{Cr}_{2} \mathrm{Cl}_{6}+2 \mathrm{KCl}+22 \mathrm{HCl}$.

Die Lösungen bereitet man folgendermaassen:

Molybdänsäurelösung. Löse $5,1044 \mathrm{~g}$ bei $120^{\circ} \mathrm{C}$. getrockneter Molybdänsäure in $200 \mathrm{cc}$ Salzsäure von 1,19 specifischem Gewicht unter Erhitzen und verdünne auf $1000 \mathrm{cc} .1 \mathrm{cc}=0,0001 \mathrm{~g} \mathrm{P}$.

Kaliumdichromatlösung. Löse $1,7474 \mathrm{~g}$ in $1000 \mathrm{cc}$ Wasser. $1 c c=0,0001 \mathrm{gP}$.

Eisenchlor ürlösung. Löse $2 g$ reinen Eisendraht in verdünnter Salzsäure und stelle die Lösung auf die Kaliumdichromatlösung.

Zinnchlor ürlösung. Löse $20 \mathrm{~g}$ in $35 \mathrm{cc}$ Salzsäure von 1,19 specifischem Gewicht und $60 \mathrm{cc}$ Wasser.

Die von dem Verfasser angeführten Beleganalysen sind zufriedenstellend, und ist vor allem die zur Ausführung erforderliche Zeit, bei Stahl 35 Minuten, bei Roheisen 1 Stunde 35 Minuten, eine sehr kurze. Ohne sonst die Methode einer weiteren Kritik unterwerfen zu wollen, verfehle ich nicht, darauf hinzuweisen, dass dieselbe insofern eine Gefahr in sich schliesst, als vorhandenes Arsen eventuell theilweise mit gefällt und als Phosphor mit bestimmt werden kann. Hz.

Zum Nachweis und zur Bestimmung von Jod, Brom und Chlor schlägt M. Dechan*) ein Verfahren vor.

Versetzt man eine concentrirte Lösung von Kaliumdichromat bei gewöhnlicher Temperatur mit Jodkalium, so findet keine oder fast keine Einwirkung statt. Erhitzt man dagegen zum Kochen, so wird alles Jod in Freiheit gesetzt nach der Gleichung:

$$
5 \mathrm{~K}_{2} \mathrm{Cr}_{2} \mathrm{O}_{7}+6 \mathrm{KJ}=3 \mathrm{~J}_{2}+8 \mathrm{~K}_{2} \mathrm{CrO}_{4}+\mathrm{Cr}_{2} \mathrm{O}_{3} \text {. }
$$

Vorhandene Bromide und Chloride bleiben unverändert. Fügt man nun nach dem Austreiben des Jods verdünnte Schwefelsäure zu, so lässt sich vorhandenes Brom gleichfalls überdestilliren.

Die Lösung von Kaliumdichromat bereitet man dem Zweck entsprechend in der Weise, dass man $40 \mathrm{~g}$ des Salzes und $100 \mathrm{cc}$ Wasser anwendet; die verdünnte Schwefelsäure stellt man her durch Mischen eines Theiles Säure von dem specifischen Gewicht 1,84 mit einem gleichen Theile Wasser. Die verdünnte Schwefelsäure gibt man in dem Verhältnisse zu, dass $8 c c$ auf $100 c c$ Wasser ențfallen, welche zur Bereitung von Kalium-

*) Journal of the chemical society $\mathbf{4 9 , 6 8 2 .}$ 